\title{
Determinação da Energia de Ativação em Hidrogéis Poliméricos a Partir de Dados Termogravimétricos
}

\author{
Marilia M. Horn, Virginia C. A. Martins, Ana M. de G. Plepis \\ Instituto de Química de São Carlos, USP
}

\begin{abstract}
Resumo: Curvas termogravimétricas com diferentes razões de aquecimento foram utilizadas para a determinação de parâmetros cinéticos seguindo o método de Flynn-Wall. Para isso, foi utilizado um hidrogel preparado a partir da mistura de dois polissacarídeos, quitosana/ xantana (QX) e outro, contendo além destes, colágeno (QXC). Os resultados mostraram que o valor de energia de ativação para o hidrogel QX foi de 3,44 kJ.mol-1 enquanto que para o QXC foi de $14,84 \mathrm{~kJ} \cdot \mathrm{mol}^{-1}$, sugerindo que a água presente no hidrogel contendo colágeno está mais fortemente ligada aos biopolímeros. Isto pode ter ocorrido devido à presença de grupos carboxílicos na estrutura colagênica.
\end{abstract}

Palavras-chave: Hidrogéis, energia de ativação, termogravimetria, biopolímeros.

\section{Determination of Activation Energy in Polymeric Hydrogels Using Thermogravimetric Analysis}

Abstract: Polyelectrolyte hydrogels formed by chitosan/xanthan (QX) and chitosan/xanthan/collagen (QXC) were prepared and thermogravimetric curves at different heating rates were obtained, with the aim of determining kinetic parameters using the Flynn-Wall method. The calculated activation energy was $3.44 \mathrm{~kJ} \cdot \mathrm{mol}^{-1}$ (QX ) and $14.84 \mathrm{~kJ}^{-\mathrm{mol}^{-1}}(\mathrm{QXC})$, suggesting stronger interactions in QXC hydrogel structure than in the QX hydrogel, probably due to the presence of carboxyl groups of collagen molecules.

Keywords: Hydrogels, activation energy, thermogravimetry, biopolymers.

\section{Introdução}

A energia de ativação, Ea, pode ser determinada utilizando curvas termogravimétricas não isotérmicas em diferentes razões de aquecimento ${ }^{[1]}$. Em polímeros esse parâmetro é determinado usualmente pelo método de Flynn-Wall ${ }^{[2]}$ que considera que a energia de ativação de um material em uma determinada perda de massa (conversão) é proporcional ao coeficiente $\mathrm{d} \beta / \mathrm{d}(1 / T)$, em que uma dependência linear é observada entre o inverso da temperatura absoluta em uma determinada porcentagem de perda de massa, 1/T, em relação ao logaritmo da razão de aquecimento, $\beta$, aplicada ${ }^{[3]}$. O método de Flynn-Wall que utiliza a termogravimetria isotérmica ou razões de aquecimento constantes é um dos métodos preferidos para a determinação da energia de ativação de polímeros, pois requer menor tempo de experimento. Entretanto, este método é limitado a decomposições simples e para polímeros que obedeçam a uma cinética de primeira ordem ${ }^{[4]}$.

Os hidrogéis são redes tridimensionais de polímeros que podem intumescer em meio aquoso e reter uma grande quantidade de água na sua estrutura ${ }^{[5]}$. Uma variedade de polímeros hidrofílicos está sendo utilizada na formação de hidrogéis para aplicação nas áreas de farmácia, medicina e biotecnologia particularmente para o tratamento de ferimentos e como suporte para liberação de fármacos ${ }^{[6]}$.

A obtenção de hidrogéis de quitosana e xantana ocorre com a formação do complexo polieletrólito por atração eletrostática entre cargas opostas presentes nos polissacarídeos. Ambos os polissacarídeos apresentam propriedades como serem biocompatíveis e biodegradáveis ${ }^{[7]}$. A adição de colágeno ao hidrogel permite a melhora da sua propriedade para, por exemplo, tratamento de queimaduras, uma vez que esta proteína tem a característica de estimular e promover o crescimento celular.

Um dos grandes interesses na utilização de hidrogéis está relacionado com o processo de liberação controlada de fármacos ${ }^{[8]}$. No caso específico de hidrogéis o intuito de se calcular a energia de ativação está relacionado ao evento de perda de água do material uma vez que a liberação ocorre via mecanismo de difusão pela solubilidade do fármaco em água. Com isso, é possível determinar o quanto de energia é necessário para romper a rede polimérica dos hidrogéis que está relacionada com a liberação controlada de fármacos ${ }^{[8]}$.

$\mathrm{Na}$ literatura são encontrados trabalhos que descrevem a determinação da energia de ativação de polímeros usando o método de Flynn-Wall ${ }^{[3,4,9]}$, entretanto, nenhum que descreva a determinação da energia de ativação em hidrogéis foi encontrado. Sendo assim, este trabalho visa à contribuição para estudos da estabilidade térmica de hidrogéis para posterior utilização como sistema de liberação de fármacos.

\section{Experimental}

\section{Materiais}

O processo de obtenção da quitosana consistiu de três etapas: desmineralização, desproteinização e desacetilação $0^{[10]}$. Para o processo de desmineralização foi utilizado 40,0 g de gládio de lula moído com $\mathrm{HCl} 0,55$ mol.L $\mathrm{L}^{-1}$ à temperatura de $25^{\circ} \mathrm{C}$ por 2 horas com agitação mecânica constante. Após isso, o material foi lavado até a neutralidade e seco a $37^{\circ} \mathrm{C}$. No processo de desproteinização o sólido obtido foi aquecido a $80{ }^{\circ} \mathrm{C}$ por 1 hora com $\mathrm{NaOH}$ $0,30 \mathrm{~mol} . \mathrm{L}^{-1}$, lavado até a neutralidade e seco em estufa, sendo obtida a $\beta$-quitina. Na última parte do procedimento obtém-se a quitosana através da desacetilação parcial dos grupos acetamida em que a $\beta$-quitina obtida é aquecida com $\mathrm{NaOH} 40 \%(\mathrm{~m} / \mathrm{m})$ a $80{ }^{\circ} \mathrm{C}$ durante 3 horas em atmosfera de nitrogênio. A partir da quitosana obtida preparou-se uma solução com concentração $0,5 \%$ em ácido acético 1,0\%. O grau de acetilação foi determinado por espectroscopia no ultravioleta ${ }^{[11]}$ e o valor encontrado foi de $10,53 \% \pm 0,03$.

A xantana utilizada foi produzida pela bactéria Xanthomonas campestris e comercializada pela Fluka - BioChemica. O material foi utilizado sem prévia purificação. A solução de xantana 
(concentração $0,5 \%$ ) foi preparada pela dissolução desta em água desionizada.

O colágeno aniônico foi obtido por tratamento de tendão bovino com uma solução alcalina contendo cloretos e sulfatos de sódio, potássio e cálcio durante 24 horas $^{[12]}$. O material foi suspenso em água desionizada e o $\mathrm{pH}$ ajustado para 3,5 com ácido acético e mantido sob refrigeração $\left(4{ }^{\circ} \mathrm{C}\right)$. $\mathrm{O}$ gel de colágeno foi preparado em concentração $0,5 \%$ em ácido acético pH 3,5. A reação de hidrólise do colágeno produz uma matriz de colágeno polianiônico carregado negativamente, a $\mathrm{pH} 7,4$, devido a hidrólise dos grupos carboxiamidas de asparaginas e glutaminas ${ }^{[13]}$.

\section{Preparação e caracterização dos hidrogéis}

Os hidrogéis foram preparados pela mistura à temperatura de $25{ }^{\circ} \mathrm{C}$ das soluções preparadas com concentração $0,5 \%$. À solução de quitosana foi adicionada a solução de xantana sob agitação sendo obtido o hidrogel quitosana:xantana (QX) em proporção 1:2 (m/m). Para a preparação do hidrogel quitosana:xantana:colágeno (QXC), a partir da mistura anterior, o colágeno foi adicionado também sob agitação em proporção 1:2:0,5 (m/m/m), respectivamente.

Os hidrogéis QX e QXC foram dialisados com tampão de Mcllavaine (fosfato dissódico - ácido cítrico) pH 5,6 durante 4 dias sob agitação constante e temperatura de $25{ }^{\circ} \mathrm{C}$. Após esse período, foram lavados com água desionizada e armazenados a $4{ }^{\circ} \mathrm{C}$ até a sua utilização.

Para a obtenção de fotomicrografias por microscopia eletrônica de varredura (MEV), os hidrogéis foram congelados e liofilizados. Posteriormente, foram colocadas nos suportes apropriados e recobertos com uma fina camada de liga ouro-paládio de $20 \mathrm{~nm}$ de espessura. A metalização foi realizada em um metalizador Balsers modelo SDC 050 e as fotomicrografias, em um microscópio da marca ZEISS modelo LEO-440.

As medidas termogravimétricas foram realizadas na razão de aquecimento de $5,10,15$ e $20{ }^{\circ} \mathrm{C} / \mathrm{min}$ em ar sintético a $80 \mathrm{~mL} / \mathrm{min}$, com amostras de aproximadamente $30 \mathrm{mg}$ em um analisador TGA-2050 da TA Instruments. Para o cálculo de energia de ativação correspondente à perda de massa nos hidrogéis foram considerados os valores de perda de massa entre 5 e $80 \%$.

\section{Resultados e Discussão}

A Figura 1 mostra as fotografias digitais dos hidrogéis obtidos, sendo que ambos apresentaram uma estrutura rígida com a manutenção da sua forma, uma coloração branca e aspecto gelatinoso.

A fotomicrografia obtida por MEV da vista lateral do complexo quitosana:xantana em um aumento de 200× (Figura 2a) revela uma estrutura do tipo folha com presença de poros, que é responsável pela retenção da água dentro da estrutura de rede ${ }^{[14]}$. Para o hidrogel QXC (Figura 2b), verifica-se que com a presença de colágeno a estrutura passa a ser mais porosa e os poros estão interconectados em todo o material em 3 dimensões, o que é extremamente importante na engenharia de regeneração tecidual, como por exemplo, para o crescimento de fibroblastos ${ }^{[15]}$.

Em um aumento maior para o hidrogel contendo colágeno (QXC) pode-se perceber claramente a presença de colágeno (indicado pelas setas) participando da estrutura do material (Figura 3).

Em um hidrogel, a água contida faz parte da estrutura do material e por essa razão estes foram submetidos a diferentes razões de aquecimento por termogravimetria com o intuito de calcular a energia de ativação correspondente ao evento de perda da água.

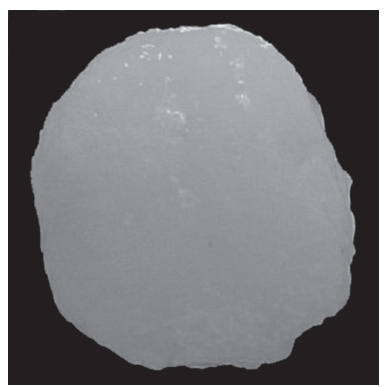

(a)

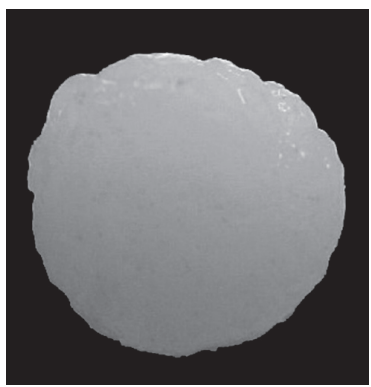

(b)
Figura 1. Fotografia digital dos hidrogéis em a) QX e b) QXC.

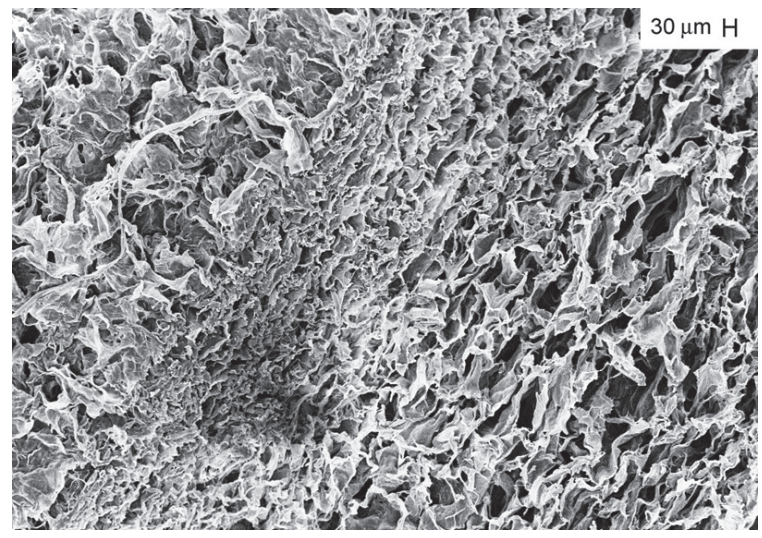

(a)

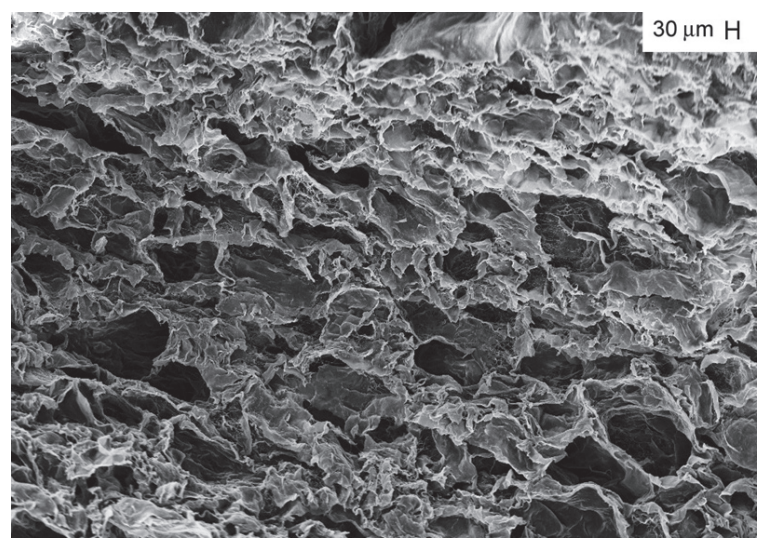

(b)

Figura 2. Fotomicrografias dos hidrogéis: a) QX12 e b) QXC12. Aumento de $200 \times$.

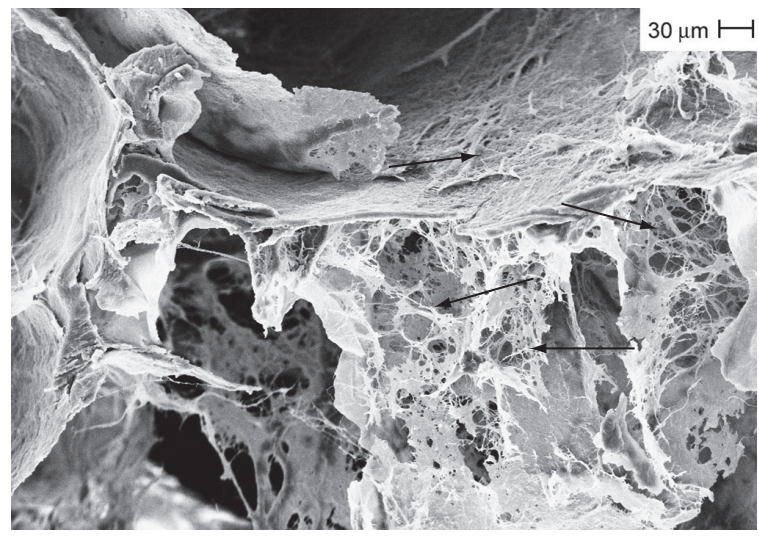

Figura 3. Fotomicrografia do hidrogel QXC. Aumento de 500×. 
Com isso, foi possível determinar o quanto de energia é necessário para romper a rede polimérica.

A Figura 4 apresenta as curvas termogravimétricas obtidas nas diferentes razões de aquecimento para os hidrogéis. As curvas mostram uma perda de massa em uma etapa, entre $25-200{ }^{\circ} \mathrm{C}$, referente à saída de água do hidrogel. Para o hidrogel QX a quantidade de água obtida foi de $98,5 \%$ e para o hidrogel QXC foi encontrado o valor de $97,7 \%$ para a razão de aquecimento de $10{ }^{\circ} \mathrm{C} / \mathrm{min}$.

O método utilizado para o cálculo foi o de Flynn e Wall ${ }^{[2]}$, que se baseia em que para um determinado valor de perda de massa o mecanismo de reação é o mesmo, independente da temperatura em que ocorra. Desta forma, é necessário determinar a temperatura em que se verifica essa porcentagem de perda de massa utilizando diferentes razões de aquecimento. Assim, o cálculo de energia de ativação a uma determinada conversão (perda de massa) pode ser feito a partir do coeficiente angular das retas obtidas em gráficos de $\ln \beta$ em função $1 / T$, conforme a Equação 1 . Os valores de perda de massa para as diferentes porcentagens, entre 5 e $80 \%$ que

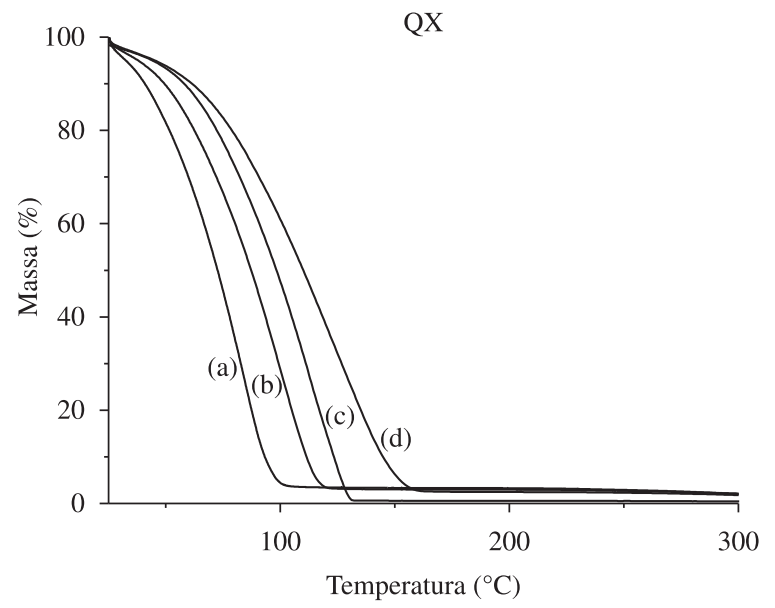

foram utilizados para o cálculo correspondem à perda de água nos hidrogéis.

$$
E a=\frac{-R}{b}\left(\frac{d \operatorname{Ln} \beta}{d(1 / T)}\right)
$$

Na Equação 1, Ea é a energia de ativação $\left(\mathrm{kJ}^{\mathrm{m}} \mathrm{mol}^{-1}\right) ; R$ é a constante dos gases $\left(8,314 \mathrm{~J} \cdot \mathrm{mol}^{-1} \cdot \mathrm{K}^{-1}\right), b$ é uma constante igual a 1 e o termo derivado, é a inclinação das curvas, $\operatorname{com} \beta$ sendo a razão de aquecimento e $T$ a temperatura absoluta $(\mathrm{K})$.

A Figura 5 apresenta as curvas de $\ln \beta$ em função de 1/T para os hidrogéis QX e QXC.

Os resultados mostram que o valor de energia de ativação para o hidrogel QX foi de 3,44 kJ.mol ${ }^{-1}$, enquanto que para o QXC foi de $14,84 \mathrm{~kJ} \cdot \mathrm{mol}^{-1}$, sugerindo que a água presente no hidrogel contendo colágeno está mais fortemente ligada aos biopolímeros. Os valores obtidos mostram que a energia necessária para a saída de água da rede do hidrogel contendo colágeno é cerca de 3 vezes maior que a necessária para o hidrogel QX.

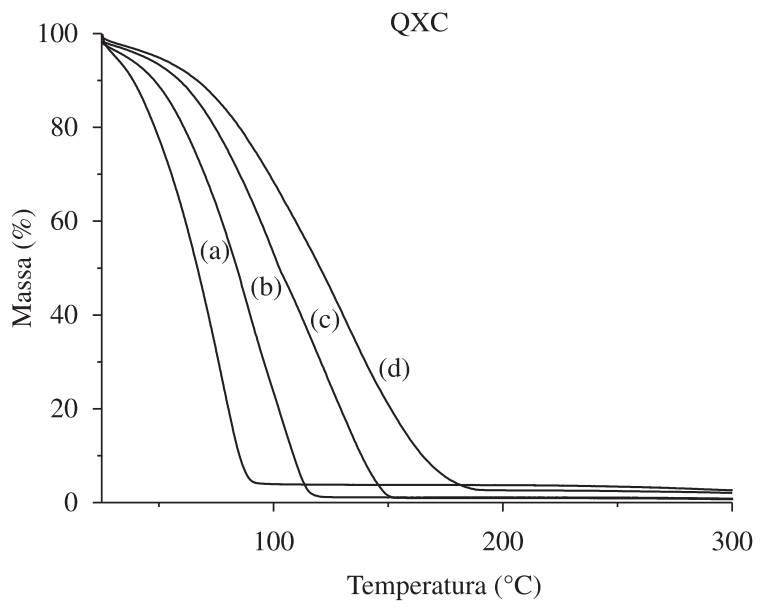
(a) $5^{\circ} \mathrm{C} / \mathrm{min}$
(b) $10{ }^{\circ} \mathrm{C} / \mathrm{min}$
(c) $15^{\circ} \mathrm{C} / \mathrm{min}$
(d) $20^{\circ} \mathrm{C} / \mathrm{min}$

Figura 4. Curvas termogravimétricas para os hidrogéis QX e QXC nas diferentes razões de aquecimento.
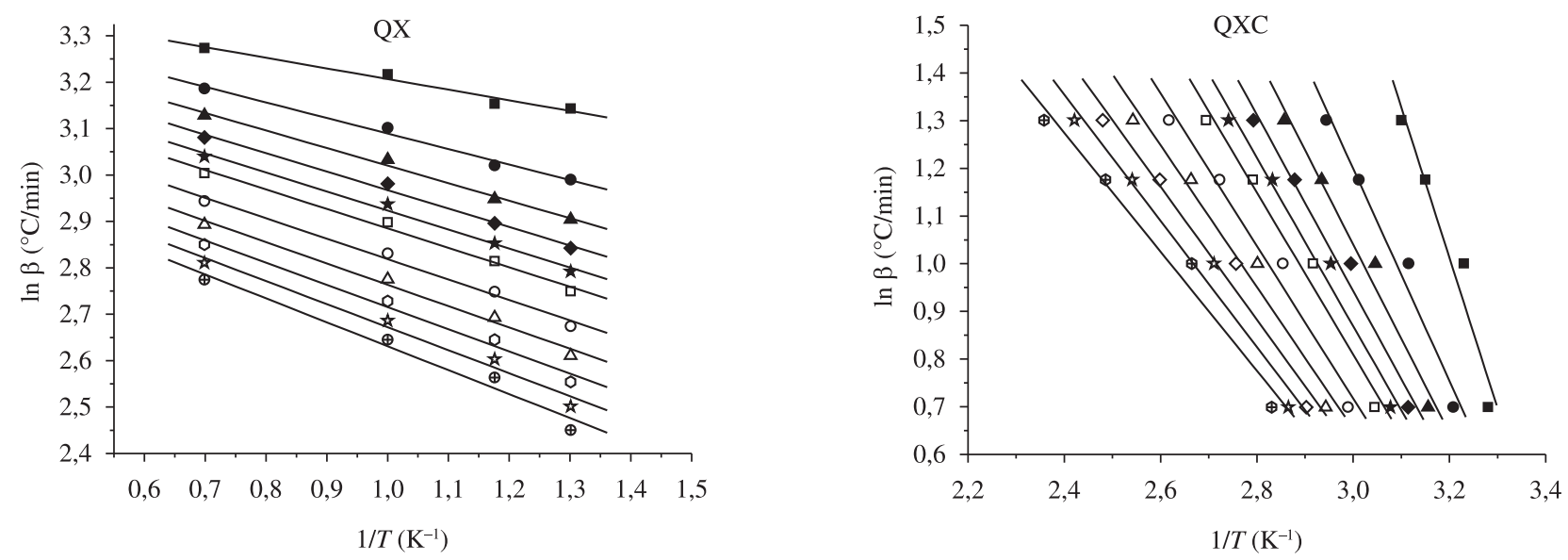

$\begin{array}{llllll}-5 \% & -10 \% & \Delta 15 \% & \star 20 \% & \star 25 \% & \text { 口 } 30 \% \\ \circ 40 \% & \Delta 50 \% & \circ 60 \% & \star 70 \% & \oplus 80 \% & \end{array}$

Figura 5. Curva de $\ln \beta$ em função de $1 / T$ para os hidrogéis QX e QXC. 


\section{Conclusão}

Os resultados obtidos mostram que o modelo cinético de primeira ordem proposto para avaliação da energia de ativação é condizente para o tipo de perda de massa que ocorre nos hidrogéis. Isso é confirmado pela linearidade observada nas curvas de logaritmo de diferentes razões de aquecimento em função do inverso da temperatura absoluta em diferentes porcentagens de conversão. A partir dos dados termogravimétricos foi verificado que a energia de ativação da amostra contendo colágeno apresentou um valor cerca de 3 vezes maior do que a amostra contendo somente quitosana e xantana. Isso sugere que a água está mais ligada provavelmente pela presença de grupos carboxílicos na molécula de tropocolágeno.

\section{Agradecimentos}

Ao Conselho Nacional de Pesquisa (CNPq) pela bolsa concedida. Ao Márcio de Paula pelas análises de microscopia eletrônica de varredura. À Miami Comércio e Exportação de Pescados Ltda. (Cananéia - SP) pelo fornecimento dos gládios de lula. À Casa de Carnes Santa Paula (São Carlos - SP) pelo fornecimento do tendão bovino.

\section{Referências Bibliográficas}

1. Fernandes, N. S.; Araújo, S. A. \& Ionashiro, M. - Eclética Química, 31, p.39-43 (2006).

2. Flynn, J. H. \& Wall, L. A. - Journal of Research of the National Bureau of Standards -A. Physics and Chemistry, 70, p.487-523 (1966).

3. Katsikas, L. \& Popovic, I. G. - The journal of physics chemistry B, 107, p.7522-7525 (2003).
4. Lage, L. G. \& Kawano, Y. - Polímeros, 9, p.82-85 (1999).

5. Peppas, N. A.; Bures, P.; Leobandung, W. \& Ichikawa, H. - European Journal of Pharmaceutics and Biopharmaceutics, 50, p.27-46 (2000).

6. Martinez-Ruvalcaba, A.; Chornet, E. \& Rodrigue, D. - Carbohydrate Polymers, 67, p.586-595 (2007).

7. Argin-Soysal, S.; Kofinas, P. \& Lo, Y. M. - Food Hydrocolloids, 23, p.202-209 (2009).

8. Barcellos, I. O.; Katime, I. A.; Soldi, V. \& Pires, A. T. N. - Polímero, 10, p.110-115 (2000).

9. Shin, S. M.; Kim, S. H. \& Song, J. K. - Macromolecular Research, 17, p.149-155 (2009).

10. Kurita, K.; Tomita, K.; Tada, T.; Ishii, S.; Nishimura, S. \& Shimoda, K. - Journal of Polymer Science: Part A Polymer Chemistry, 31, p.485-491 (1993).

11. Liu, D.; Wei, Y.; Yao, P. \& Jiang, L. - Carbohydrate Research, 341, p.782-785 (2006).

12. Lacerda, C.; Plepis, A. M. G. \& Goissis,G. - Química Nova, 21, p.267-271 (1998).

13. Ogawa, C. A. \& Plepis, A. M. G. - Polímeros, 12, p.115-122 (2002).

14. Higuchi, C. I. - "Chitosan-Xanthan Hydrogel: a matrix of inclusion and the delivery of drugs", Tese de Doutorado, Département de génie chimique, Université de Sherbrooke, Québec, Canadá (2002).

15. O’Brien, F. J.; Harley, B. A.; Yannas, I. V. \& Gibson, L. J. - Biomaterials, 26, p.433-441 (2005).

Enviado: 07/10/09 Reenviado: 18/12/09

Aceito: $22 / 02 / 10$

DOI: $10.1590 / \mathrm{S} 0104-14282010005000025$ 